\author{
P. M. Catalano $\cdot$ M. Hoegh $\cdot$ J. Minium $\cdot$ \\ L. Huston-Presley $\cdot$ S. Bernard $\cdot$ S. Kalhan • \\ S. Hauguel-De Mouzon
}

\title{
Adiponectin in human pregnancy: implications for regulation of glucose and lipid metabolism
}

Received: 6 December 2005 / Accepted: 27 February 2006 / Published online: 11 May 2006

C) Springer-Verlag 2006

\begin{abstract}
Aims/hypothesis: Adiponectin is upregulated during adipogenesis and downregulated in insulin-resistant states. The mechanism(s) governing the re-arrangements from adipogenesis to facilitated lipolysis during pregnancy are unknown. Our purpose was to analyse the role of adiponectin relative to the metabolic changes in human pregnancy. Subjects, materials and methods: Lean women $\left(\right.$ BMI $<25 \mathrm{~kg} / \mathrm{m}^{2}$ ) were evaluated longitudinally before conception, and in early (12-14 weeks) and late (34-36 weeks) pregnancy. Insulin sensitivity was measured using the glucose clamp technique. Venous blood and subcutaneous adipose tissue biopsies were obtained at each time point. Results: Adiponectin concentrations were lower in the third trimester than in the pregravid condition $(9.9 \pm 1.4$ vs $13.5 \pm 1.8 \mu \mathrm{g} / \mathrm{ml}$ ). The hypoadiponectinaemia was reflected by a 2.5 -fold decrease in white adipose tissue adiponectin mRNA. These changes were associated with a $25 \%$ increase in fat mass $(23.7 \pm 2.9 \mathrm{vs} 18.9 \pm 2.9 \mathrm{~kg})$. Insulin infusion decreased high molecular weight adiponectin complexes in pregravid women ( $9.9 \pm 0.6$ vs $6.2 \pm 0.06)$ and the suppressive effect of insulin was lost during pregnancy. The pregnancy-mediated changes in adiponectin were strongly correlated with basal insulin levels and insulin sensitivity $(p<0.0001)$. The relationship between adipo-
\end{abstract}

P. M. Catalano · M. Hoegh · J. Minium · L. Huston-Presley · S. Hauguel-De Mouzon $(\square)$

Department of Reproductive Biology,

Case Western Reserve University,

2550 MetroHealth Drive,

Cleveland, OH 44109, USA

e-mail: shdemouzon@metrohealth.org

Tel.: +1216-778-3148

Fax: +1216-778-1574

S. Bernard

Department of Plastic Surgery,

Case Western Reserve University,

Cleveland, $\mathrm{OH}$, USA

S. Kalhan

Schwartz Center for Nutrition and Metabolism,

MetroHealth Medical Center,

Cleveland, $\mathrm{OH}$, USA nectin and insulin sensitivity was related to the decreased insulin regulation of glucose utilisation $(r=0.55, p<0.001)$ but not of endogenous hepatic glucose production. Conclusions/interpretation: These data demonstrate that pregnancy is associated with adiponectin changes in lean women. Hypoadiponectinaemia is reflected by a lower amount of high molecular weight adiponectin and by the ratio of high to low molecular weight multimers. The adiponectin changes relate to decreased insulin sensitivity of glucose disposal rather than alterations of lipid metabolism.

Keywords Adiponectin multimers - Glucose production . Glucose utilisation · Human · Insulin sensitivity · Lipid oxidation · Pregnancy · White adipose tissue

Abbreviations E: Early gestation - GDM: Gestational diabetes mellitus - HMW: High molecular weight adiponectin complex $\cdot \mathrm{L}$ : Late gestation $\cdot$ LMW: Low molecular weight adiponectin complex $\cdot \mathrm{P}$ : Before conception $\cdot$ RQ: Respiratory quotient

\section{Introduction}

To meet the energy requirements of fetal development during pregnancy dynamic re-arrangements of adipose tissue, associated with an initial period of adipogenesis followed by increased lipolytic activity, are required $[1,2]$. In humans, these changes are facilitated by decreased insulin sensitivity, primarily at the peripheral level and secondarily at the hepatic level, occurring in late pregnancy [3]. The blunted effect of insulin on skeletal muscle and white adipose tissue allows decreased glucose utilisation by maternal peripheral tissues and increased availability to the feto-placental unit [4]. The molecular mechanism(s) governing the integrated alterations of lipid metabolism from adipogenesis to facilitated lipolysis are unknown.

Recent studies suggest that the regulation of the lipid stores is tightly regulated by adipocyte-specific proteins, referred to as adipocytokines or adipokines, that interfere 
with insulin action. Among these, the current view is that TNF- $\alpha$, interleukin- 6 and resistin negatively regulate insulin action, in contrast to the insulin-sensitising action of leptin and adiponectin [5]. Adiponectin is the most abundant adipocyte-derived product identified to date, with circulating concentrations in the nanomolar range [6]. Adipocytes secrete adiponectin as multimeric complexes with a high molecular weight oligomer (HMW) and a low molecular weight hexamer (LMW), which account for the majority of systemic circulating adiponectin [7]. Unlike for other adipokines, plasma adiponectin concentrations are inversely correlated with adiposity in humans $[8,9]$. In addition, total adiponectin is positively correlated with insulin sensitivity in human and rodents $[10,11]$. In obesity and diabetes, low plasma adiponectin concentrations are related to the degree of hyperinsulinaemia and the severity of insulin resistance [11-13]. Adiponectin and HMW adiponectin levels are negatively correlated with serum triglycerides and positively with HDL particles [14, 15]. The mechanisms mediating adiponectin-lipid interactions remain unknown but it is suggested that HMW adiponectin plays a prominent role in the metabolic changes $[15,16]$. As a putative signal generated by white adipose tissue and modulating insulin sensitivity in relation to lipid metabolism, adiponectin emerged as an interesting candidate for controlling homeostasis of the lipid metabolism during pregnancy. To better understand the role of adiponectin in human pregnancy metabolism, we performed a longitudinal study of adiponectin gene and protein expression in relation to measures of the insulin sensitivity of glucose and lipid metabolism in women with normal glucose tolerance. We show that the absolute amount of HMW as well as the ratio of HMW to LMW adiponectin multimers is decreased during pregnancy, as reflected by systemic hypoadiponectinaemia.

\section{Subjects, materials and methods}

\section{Clinical study protocol}

The study was conducted in the General Clinical Research Center (GCRC) at MetroHealth Medical Center, Case Western Reserve University. The study protocol was approved by the Hospital Institutional Review Board and by the Scientific Review Committee of the GCRC. Written informed consent was obtained from each subject before evaluation. Ten healthy women were recruited before a planned pregnancy to participate in this study. None of the subjects was using hormonal contraception, tobacco, or other medications that might affect carbohydrate metabolism or energy expenditure. None of the study subjects had diabetes mellitus before conception based on the results of a 100-g oral glucose tolerance test [17]. All patients were screened for gestational diabetes mellitus (GDM) between 20 and 24 weeks pregnancy with a 1 -h 50 -g glucose test. If positive, GDM was ruled out with a 100-g OGTT. All subjects were normotensive throughout pregnancy and none developed medical or obstetrical complications. All subjects were planning to conceive as soon as the baseline pregravid studies were completed. Each subject was evaluated before conception $(\mathrm{P})$, in early gestation $(\mathrm{E} ; 12-14$ weeks) and again in late gestation (L; 34-36 weeks). Pregravid subjects were evaluated in the follicular phase of the menstrual cycle. Each subject was instructed in a standard dietary regimen 2 weeks before each study period by the GCRC nutritionist to maintain weight before conception.

\section{Anthropometric measurements}

Percentage of body fat was calculated according to the equations of Keys and Brozek [18] to estimate fat-free mass at times $\mathrm{P}$ and $\mathrm{E}$. At time L, body fat was calculated according to the equations of Catalano et al. [1], adjusting the decreased density of fat-free mass because of the increased total body water in late pregnancy. In addition, skinfold thickness measurements were obtained at seven sites: triceps, biceps, subscapular, subcostal, suprailiac, mid- and lower thigh, with a Harpenden skinfold calliper (British Indicators, Sussex, UK). All measurements were obtained on the left side by one examiner (P. M. Catalano).

Hyperinsulinaemic-euglycaemic clamp and analytical procedures

Insulin sensitivity in skeletal muscle, liver and fat was determined by insulin-mediated whole-body glucose disposal, suppression of hepatic glucose output evaluated during a hyperinsulinaemic-euglycaemic clamp, and suppression of NEFA concentrations and rate of lipid oxidation by indirect calorimetry. Basal endogenous glucose production (primarily hepatic) was estimated after an 11-h overnight fast using a primed constant infusion of $\left[6,6^{2} \mathrm{H}_{2}\right]$ glucose. Basal glucose turnover was estimated according to the steady-state equations of Steele [19]. Insulin sensitivity was estimated as the glucose infusion rate required to maintain euglycaemia during the clamp plus any residual endogenous glucose production during insulin infusion. The 2-h hyperinsulinaemic-euglycaemic clamp was performed after estimating basal endogenous glucose production [20] to estimate peripheral insulin sensitivity. Insulin was infused at $25 \mathrm{mU} \cdot \mathrm{m}^{-2} \cdot \mathrm{min}^{-1}$ to achieve plasma insulin concentrations of approximately $483 \mathrm{pmol} / \mathrm{l}$. During the last $40 \mathrm{~min}$ of insulin infusion, blood samples were obtained at 5-min intervals to measure plasma glucose, insulin and adiponectin. The amount of glucose infused was calculated for each 10-min period and averaged for the last $40 \mathrm{~min}$ of the 2 -h glucose infusion. This value was used to estimate the glucose disposal as glucose uptake in peripheral tissues under steady-state conditions.

Residual endogenous glucose production during insulin infusion was estimated by maintaining the constant infusion of labelled glucose and by adding additional isotope to the $20 \%$ glucose infusion as described [21]. Plasma enrichment was determined with a gas chromatograph mass spectrometer (model 5985B; Hewlett-Packard, 
Palo Alto, CA, USA). The insulin sensitivity index was calculated by dividing the glucose infusion rate by the mean insulin concentration achieved during the clamp.

Metabolic rate and route of glucose disposal

The metabolic rate and route of glucose disposal were estimated by indirect calorimetry at steady-state (during the last $40 \mathrm{~min}$ of both the basal endogenous glucose production and the 2-h hyperinsulinaemic-euglycaemic clamp). A ventilated hood system was used for the continuous collection and mixing of expired air as previously described. The total volume of expired air was corrected for standard temperature and pressure conditions. The electrical outputs were interfaced with a desktop computer and integrated measurements of oxygen consumption $\left(\mathrm{VO}_{2}\right.$, measured in $\left.\mathrm{ml} / \mathrm{min}\right)$ and carbon dioxide production $(\mathrm{ml} / \mathrm{min})$ were made; the respiratory quotient (RQ) was averaged and recorded every $5 \mathrm{~min}$. All the studies were conducted at the same time of day during each period of study. All subjects remained for at least $2 \mathrm{~h}$ in a semi-recumbent position on their left side (so as not to impair venous return in late gestation) before the indirect calorimetry measurements.

The quantity of urinary urea nitrogen excreted during the study was used as an index of protein oxidation, assuming that $1 \mathrm{~g}$ of nitrogen is equivalent to $6.25 \mathrm{~g}$ of protein, and to calculate non-protein RQ. Oxidation rates of carbohydrate and lipid were then calculated according to the tables of Frayn [22]. The non-oxidative route of glucose disposal (i.e. glucose stored as glycogen, conversion to three-carbon fragments and lipogenesis) was determined in the basal state as the difference between total basal glucose turnover and rate of glucose oxidation as estimated from indirect calorimetry. Non-oxidative glucose disposal during the clamp was estimated as the difference between total glucose disposal during the euglycaemic clamp studies and rate of glucose oxidation as estimated from indirect calorimetry. RQ $>1.0$ was assumed to represent lipogenesis. The ${ }^{2} \mathrm{H}$-enrichment of glucose was measured as previously described [21].

\section{Analytical procedures}

Plasma samples were frozen immediately after separation and stored at $-20^{\circ} \mathrm{C}$. Blood glucose was determined by the glucose oxidase method. Plasma insulin was measured by RIA (Linco, St Charles, MO, USA), NEFA concentrations were determined with an enzymatic method (WAKO, Richmond, VA, USA) and total plasma adiponectin concentrations were measured by ELISA (Linco).

White adipose tissue biopsies

Subcutaneous white adipose tissue was obtained from the lateral gluteal region using an open-ended liposuction biopsy needle catheter. Biopsies were obtained in the GCRC after an overnight fast. Part of the gluteal region was prepared with betadine and alcohol, and sterile drapes were placed. Approximately $6 \mathrm{ml}$ of $2 \%$ lidocaine was injected into the subject's skin with a 25 -gauge needle. After $10 \mathrm{~min}$ the skin was nicked with a \#11 blade to insert the biopsy needle. Suction was then applied using a $60-\mathrm{cm}^{3}$ syringe and tygon tubing to ensure that the adipose tissue was drawn into the hollow of the needle. The sleeve was then rotated and the procedure repeated two or three more times. Approximately $3-5 \mathrm{~g}$ of adipose tissue was obtained.

\section{SDS-PAGE and immunoblotting}

SDS-PAGE was performed according to the standard Laemmli procedure [23]. Equal volumes of plasma samples $(1 \mu \mathrm{l})$ were solubilised in $5 \times$ Laemmli buffer and directly loaded on a $8-16 \%$ Tris-glycine polyacrylamide gel for separation of adiponectin complexes under nondenaturing conditions. Alternatively, samples were heated at $100^{\circ} \mathrm{C}$ for $5 \mathrm{~min}$ in the presence of $100 \mathrm{mmol} / \mathrm{l}$ dithiothreitol for complete reduction. Proteins separated by SDS-PAGE were transferred onto a nitrocellulose membrane (Hybond ECL; Amersham Biosciences, Piscataway, NJ, USA). The blot was stained with $0.1 \%$ Ponceau solution to confirm transfer. After blocking for $1 \mathrm{~h}$ in $5 \%$ milk solution (Blotto; Santa Cruz Biotechnology, Santa Cruz, CA, USA) the blots were incubated with antiadiponectin antibody, final concentration $3 \mu \mathrm{g} / \mathrm{ml}$ (Chemicon, Temecula, CA, USA), in $2 \%$ milk solution overnight, then incubated with horseradish peroxidase-conjugated anti-rabbit antibody (Chemicon) 1:5,000 in 2\% milk for 1 h. After thorough washing, immunoreactive signals were visualised with ECL Western blotting detection reagent (Amersham Biosciences) and quantified by densitometry (GS-670; Bio-Rad, Hercules, CA, USA).

\section{Real-time PCR}

RNA was purified from white adipose tissue (approximately $300 \mathrm{mg}$ ) using Trizol with the following alterations to the manufacturer's recommendations: $4 \mathrm{ml}$ Trizol per $250 \mathrm{mg}$ tissue was used, and following homogenisation all procedures were performed on ice. The cDNA was synthesised using Superscript II RNase H Reverse Transcriptase Kit (Invitrogen, Carlsbad, CA, USA) according to the manufacturer's instructions. Adiponectin, and actin gene expression were measured by real-time PCR using a LightCycler FastStart DNA Master SYBR Green I kit (Roche, Indianapolis, IN, USA). All PCR mixtures contained $2 \mathrm{mmol} / \mathrm{l} \mathrm{MgCl}_{2}$ and all primers were used at a final concentration of $0.2 \mu \mathrm{mol} / 1$ each. Absolute copy numbers were calculated from a genomic DNA standard curve created from a series of tenfold dilutions. Results were normalised for actin expression. 
Statistical analysis

The data are expressed as mean \pm standard error (SD). Statistical analyses were performed using $t$-tests with analysis of variance with repeated measures for two groups to determine changes over time. Linear and non-linear regression analyses were used to assess the correlation between independent variables. Statistical analysis was performed with the Statview II (SAS Institute Version 5, Cary, NC, USA) statistical package. Values of $p \leq 0.05$ were considered significant.

\section{Results}

Longitudinal changes of adiponectin in normal pregnancy

To determine the basis for adiponectin regulation during pregnancy, we performed longitudinal measurements in individual subjects before and at two time-points during pregnancy. Metabolic and anthropometric characteristics of the subjects are given in Table 1. The physiological adaptations of maternal metabolism to pregnancy included a progressive increase in total fat mass with a significant increase of $4.8 \mathrm{~kg}$ by the third trimester. The decrease of fasting glucose was concomitant with an increase in fasting plasma insulin. These changes were indicative of loss of insulin sensitivity in late pregnancy, as shown by the decrease in the insulin sensitivity index (Table 2).

Total plasma adiponectin was decreased in late pregnancy compared with pregravid measurements $(9.9 \pm 1.4 \mathrm{vS}$ $13.5 \pm 1.8 \mu \mathrm{g} / \mathrm{ml}, p<0.004)$. This was associated with a 2.5 fold reduction of adiponectin mRNA levels in white adipose tissue (Fig. 1). Both plasma adiponectin concentrations and mRNA levels were negatively correlated with fat mass $(r=-0.50, p=0.005)$. The absolute amount of adiponectin multimers was quantified by non-denaturing gradient gel electrophoresis, which allows adequate separation of HMW and LMW complexes [16, 24]. The primary forms of circulating adiponectin were a HMW oligomer of approximately $340 \mathrm{kDa}$, corresponding to a multimeric assembly of $12-16$ molecules, and a LMW

Table 1 Anthropometric and metabolic characteristics of study population

\begin{tabular}{lccl}
\hline & Pregravid & $\begin{array}{l}\text { Early } \\
\text { pregnancy }\end{array}$ & $\begin{array}{l}\text { Late } \\
\text { pregnancy }\end{array}$ \\
\hline $\begin{array}{l}\text { Gestational age } \\
\quad(\text { weeks) }\end{array}$ & & $12.9 \pm 0.3^{\mathrm{a}}$ & $35.2 \pm 0.0^{\mathrm{a}}$ \\
BMI $\left(\mathrm{kg} / \mathrm{m}^{2}\right)$ & $23.3 \pm 1.4$ & $24.0 \pm 1.4$ & $28.7 \pm 1.2^{\mathrm{a}}$ \\
Fat mass $(\mathrm{kg})$ & $18.9 \pm 2.9$ & $19.9 \pm 2.6$ & $23.7 \pm 2.9^{\mathrm{a}}$ \\
Body fat $(\%)$ & $27.6 \pm 7.6$ & $28.4 \pm 2.0$ & $28.3 \pm 2.1$ \\
Fat-free mass $(\mathrm{kg})$ & $46.9 \pm 2.5$ & $48.0 \pm 1.9$ & $57.5 \pm 1.8^{\mathrm{a}}$ \\
\hline
\end{tabular}

Results are mean \pm SD of ten subjects studied longitudinally Differences are statistically significant compared with pregravid with ${ }^{\mathrm{a}} p<0.001$ hexamer of approximately 160-180 kDa (Fig. 2a). Under reducing conditions the multimeric circulating forms could be converted to a 30-kDa monomer (Fig. 2a). The absolute amount of both HMW and LMW adiponectin multimers decreased with advancing pregnancy, but only the change in HMW was significant $(p<0.05)$ in late pregnancy compared with pregravid (Fig. $2 \mathrm{~b}$ ). The change in the complex distribution from pregravid to late pregnancy was also significant when expressed as the HMW:LMV ratio $(10.1 \pm 0.4$ vs $6.1 \pm 0.3, p<0.01)$ or as the HMW/(HMW+LMW) adiponectin index $(15.3 \pm 0.03 \mathrm{vs}$ $7.2 \pm 0.02, p<0.03)$.

\section{Adiponectin and insulin effect}

The effect of insulin on adiponectin regulation was evaluated during a pancreatic clamp with a 2 -h insulin infusion increasing insulin plasma levels by approximately five- to sixfold over fasting levels (average at steady state: $485 \pm 34 \mathrm{pmol} / \mathrm{l})$. Insulin infusion decreased total adiponectin $(13.5 \pm 1.8 \mathrm{vs} 10.0 \pm 1.2 \mu \mathrm{U} / \mathrm{ml}, p<0.01)$ as well as the percentage of HMW:LMW complexes $(9.9 \pm 0.6$ vs $6.2 \pm 0.06, p<0.05$ ) in pregravid individuals (Fig. 3). The suppressive effect of insulin on adiponectin was not observed in early or late pregnancy, indicating that insulin action differs in the pregnant and non-pregnant states. There was a strong negative correlation between fasting insulin and adiponectin concentration throughout pregnancy $(r=-0.66, p<0.0001)$ and the correlation remained significant $(r=-0.70, p<0.0001)$ when the data for late pregnancy were excluded from the analysis (Fig. 4).

To further investigate the relationship between impairment of insulin action and adiponectin regulation, we correlated specific measures of insulin sensitivity performed under insulin-stimulated conditions with plasma adiponectin concentrations in pregravid and pregnant subjects. A significant correlation $(r=0.55, p=0.001)$ was observed between the rate of glucose disposal $R_{\mathrm{d}}$, taken as an index of insulin sensitivity, and plasma adiponectin (Fig. $5 a)$. The correlation was lost $(r=0.41, p=0.08)$ when the data taken during late pregnancy were omitted from the analysis (Fig. 5b), suggesting that the changes in peripheral insulin action are instrumental in the downregulation of adiponectin.

\section{Adiponectin and energy metabolism}

Insulin was less potent in the suppression of endogenous glucose production in late pregnancy compared with both early pregnancy and the pregravid condition (Table 2). Changes in adiponectin concentration were not related to endogenous glucose production either under basal conditions $(r=0.13,0.11$ and 0.30$)$ or insulin-stimulated conditions $(r=-0.12,-0.04,-0.03)$ at pregravid, early and late times, respectively. Based on controversial reports debating whether the regulation of lipid oxidation in muscle is a primary target of adiponectin action, we next examined the 
Table 2 Measures of glucose metabolism under basal and hyperinsulinaemic-euglycaemic steady-state conditions

\begin{tabular}{|c|c|c|c|}
\hline & Pregravid & Early pregnancy & Late pregnancy \\
\hline $\begin{array}{l}\text { Fasting glucose } \\
(\mathrm{mmol} / \mathrm{l})\end{array}$ & $4.9 \pm 0.1$ & $4.5 \pm 0.1^{\mathrm{b}}$ & $4.2 \pm 0.1^{\mathrm{a}}$ \\
\hline $\begin{array}{l}\text { Fasting insulin } \\
(\mathrm{pmol} / \mathrm{l})\end{array}$ & $90.9 \pm 7.6$ & $73.6 \pm 7.6$ & $114.6 \pm 7.6^{\mathrm{a}}$ \\
\hline $\begin{array}{l}\text { ISI }\left(\mathrm{mg} \cdot \mathrm{kg}^{-1} \cdot \min ^{-1}\right) \\
\operatorname{HGP}\left(\mathrm{mg} \cdot \mathrm{kg}^{-1} \cdot \mathrm{min}^{-1}\right)\end{array}$ & $0.14 \pm 0.04$ & $0.15 \pm 0.05$ & $0.08 \pm 0.02^{\mathrm{a}}$ \\
\hline Basal & $2.5 \pm 0.07$ & $2.5 \pm 0.06$ & $2.7 \pm 0.1$ \\
\hline Insulin clamp & $0.06 \pm 0.2^{\mathrm{b}}$ & $0.05 \pm 0.02^{\mathrm{b}}$ & $0.09 \pm 0.3^{\mathrm{a}, \mathrm{b}}$ \\
\hline
\end{tabular}

Results are means \pm SD of ten subjects studied longitudinally before and during pregnancy. Differences statistically significant compared to non-pregnant with $p<0.001$

ISI Insulin sensitivity index, HGP hepatic glucose production

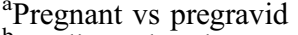

${ }^{\mathrm{b}}$ Insulin vs basal

relationship between fat oxidation and adiponectin levels. The insulin-resistant state of lipid metabolism in late pregnancy was clearly reflected by the attenuated ability of insulin to inhibit fat oxidation (Table 3). However, the changes in lipid oxidation under insulin-stimulated conditions were not correlated with the changes in plasma adiponectin whether they were examined in the pregravid period, or in early or late pregnancy $(r=0.20, p=0.60$; $r=-0.19, p=0.60 ; r=-0.37, p=0.66)$. The significant increase in circulating NEFA under insulin-stimulated conditions indicated that insulin was less efficient at suppressing NEFA release from white adipose tissue in late pregnancy compared with pregravid women, hence facilitating lipolysis (Table 3 ). When analysed throughout pregnancy, the insulin-induced changes in NEFA were independent of adiponectin concentrations $(p=0.54,0.61,052)$.

\section{Discussion}

Pregnancy is a situation of hypoadiponectinaemia

Previous studies have shown that hypoadiponectinaemia is a hallmark of situations where insulin resistance occurs, including obesity, type 2 diabetes and gestational diabetes
$[12,24-26]$. Our longitudinal analysis in women from the pregravid state to the third trimester indicates that pregnancy in humans is a physiological situation in which circulating adiponectin is decreased, as is found in mice $[11,27]$. The $25 \%$ increase in fat mass from pregravid state to late pregnancy, together with the negative correlation between circulating adiponectin concentrations and fat mass, suggests that adipose tissue accretion is associated with signals for lowering adiponectin production or secretion even in the absence of obesity. Other situations of hypoadiponectinaemia, such as lipodystrophy and liver disease, support the view that a re-distribution of adipose tissue is critical in lowering adiponectin levels [28, 29]. This may also be relevant in pregnancy when fat accretion takes place predominantly in the central compartment [30]. In addition to absolute changes in fat mass, there is also a negative relationship between adiponectin and intracellular lipid content in muscle and liver [31]. However, this relationship may reflect insulin resistance as well as increased lipid content per se because intracellular accumulation of lipids in non-adipose tissues is a process aimed at buffering excess circulating NEFA. In late pregnancy, this may be supported by the decreased ability of insulin to suppress lipolysis compared with early pregnancy (Table 3).

\section{Regulation of adiponectin expression}

The parallel changes in total plasma adiponectin and adipose tissue adiponectin gene expression argue in favour of plasma adiponectin levels reflecting pregnancy-associated adiponectin production. Whether the regulation is at the transcriptional or translational level or at the level of secretion into the systemic circulation is not known. Very few data are available concerning the transcriptional regulation of the adiponectin gene. A c/EBP- $\alpha$ consensus binding site has been found in the first intron of the gene but functional significance for regulation is not yet established [32]. Glucocorticoids, TNF- $\alpha$ or IL-6 are putative negative regulatory factors of the adiponectin gene whereas insulin-mediated regulation has not been documented $[33,34]$. This may be surprising in view of the observation that insulin is able to suppress the concentra-
Fig. 1 Longitudinal changes in adiponectin during pregnancy. a Total plasma adiponectin concentration measured by ELISA. b White adipose tissue (WAT) mRNA levels measured by real-time RT-PCR. $P$, pre-gravid; $E, 12-14$ weeks of pregnancy; $L, 36-38$ weeks of pregnancy. Results are mean \pm SD. ${ }^{*} p<0.05$ vs non-pregnant
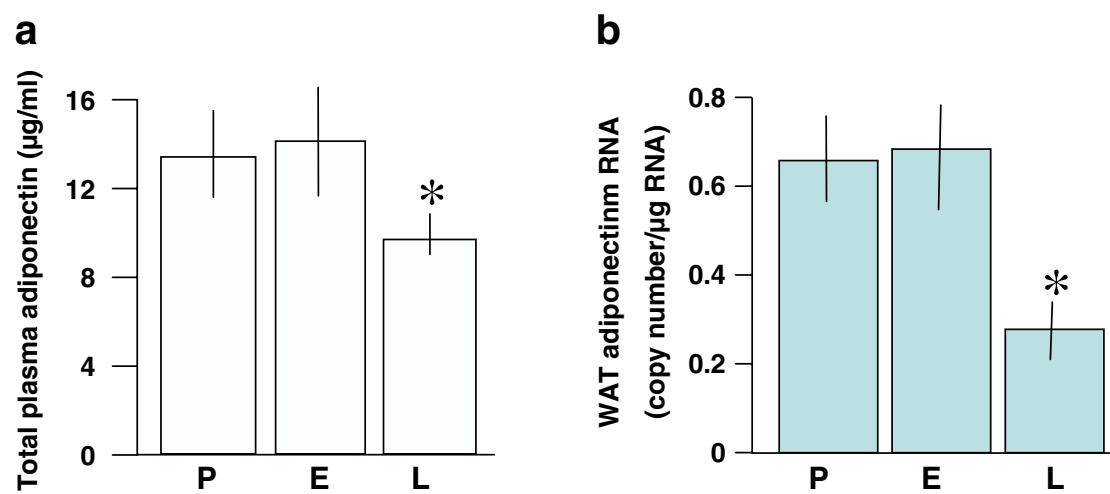

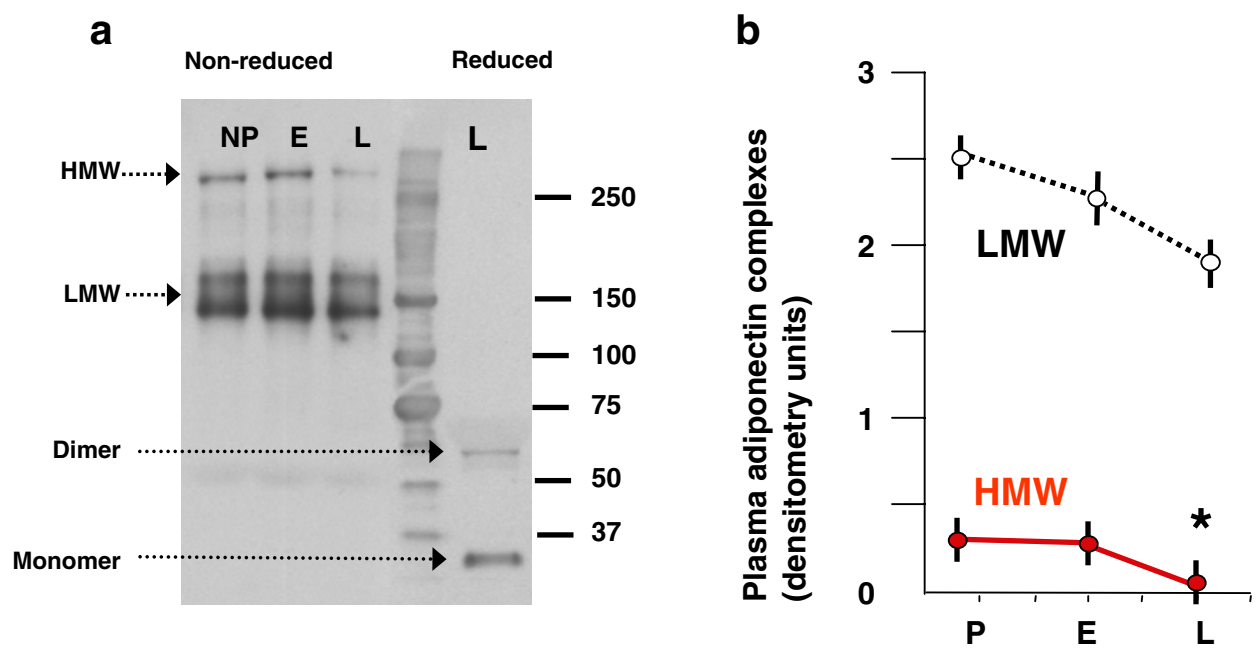

Fig. 2 Longitudinal changes in adiponectin complex distribution. a Representative Western blot showing high molecular weight oligomers $(H M W)$ migrating at approximately $340 \mathrm{kDa}$ and low molecular weight hexamers $(L M W)$ migrating at $130-160 \mathrm{kDa}$ under non-reducing and non-denaturing SDS-PAGE conditions. b Distribution of HMW and LMW adiponectin complexes quantified by densitometry after $1 \mu l$ plasma samples were subjected to SDS-PAGE as shown in (a). Data are means \pm SD $(n=8)$. $P$, pre-gravid; $E, 12-14$ weeks of pregnancy; $L, 36-38$ weeks of pregnancy. ${ }^{*} p<0.05$ vs non-pregnant tion of plasma adiponectin. Total adiponectin, as well as HMW multimers, decrease in response to insulin infusion $[6,35,36]$, but this may be regulated at the level of secretion rather than transcription [37]. Adiponectin is secreted as HMW and LMW multimeric complexes, and the amount of circulating complexes is likely to be regulated at the tissue level because LMW and HMW complexes do not interchange in the serum [38]. This process is supported by our findings that the lowering in total adiponectin in late pregnancy is reflected primarily at the level of HMW complexes (Fig. 2), although HMW represents $<15 \%$ of total circulating adiponectin. This supports previous reports suggesting that HMW adiponectin is the active form of the protein $[7,16,38]$.

Different adiponectin oligomers activate different transduction pathways and, this may account for regulating the

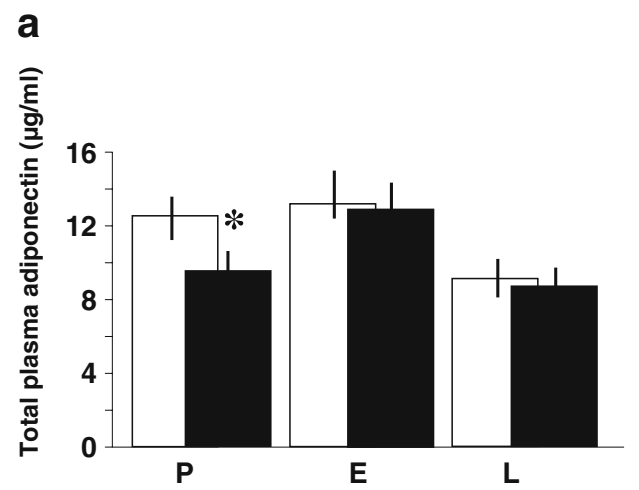

Fig. 3 Effect of insulin on total plasma adiponectin and adiponectin complex distribution. Plasma was obtained in basal state (overnight fast) and under steady-state conditions during euglycaemichyperinsulinaemic clamp (25 mU insulin $\mathrm{kg}^{-1} \mathrm{~m}^{-2}$ ) in longitudinal studies of women who were pre-gravid $(P)$, in early $(E)$ and late $(P)$ pregnancy. a Total adiponectin. Open bars, basal; filled bars, bioactivity of adiponectin in relation to insulin action [39]. The loss of insulin effect on adiponectin expression during pregnancy compared with the non-gravid state indicates an impairment with insulin action in white adipose tissue, the main site of adiponectin production. This may result from increased levels of insulin antagonists because there is no apparent intrinsic defect of insulin receptor activity in adipocytes from pregnant women [40].

\section{Functional significance of hypoadiponectinaemia}

Adiponectin is the only adipocyte-derived hormone to be down-regulated in insulin-resistant states. It has been extensively debated whether impaired insulin action is the cause or consequence of adiponectinaemia. Our observa-

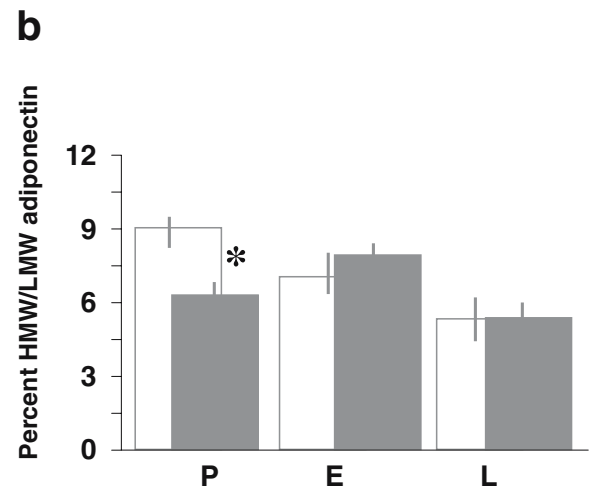

insulin. b Adiponectin complex distribution quantified by SDSPAGE of $1 \mu \mathrm{l}$ serum samples (as shown in Fig. 2) and expressed as percent oligomeric HMW components/hexameric LMW components. Open bars, basal; shaded bars, insulin. ${ }^{*} p<0.05$ insulin vs basal 


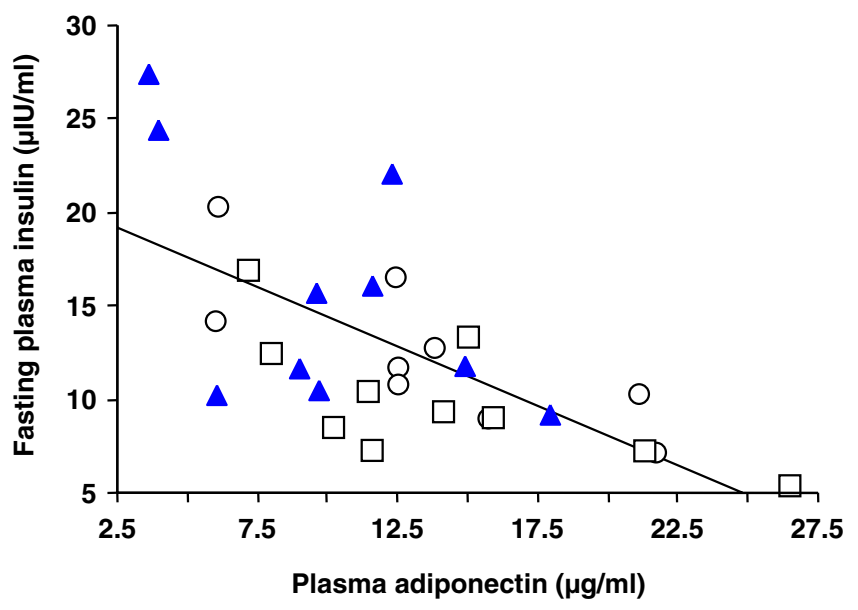

Fig. 4 Fasting insulin concentration in relation to adiponectin during pregnancy. Fasting insulin concentrations correlated with total plasma adiponectin. Circles, pre-gravid; squares, early pregnancy; triangles, late pregnancy. $r^{2}=0.43 ; y=20.7-0.001 x ; R=-0.66$; $p=0.0001$

tion that adiponectin is correlated with glucose disposal in late pregnancy is in agreement with studies suggesting that adiponectin is implicated in the regulation of muscle glucose utilisation, whether it is mediated through decreased glucose uptake, AMP protein kinase phosphorylation or insulin signalling [41]. Our results suggest that systemic adiponectin specifically predicts the insulin sensitivity of glucose but not lipid during pregnancy.

Correlative studies have suggested functional interactions between circulating NEFA and adiponectin but it is not yet clarified whether NEFA regulate adiponectin or adiponectin regulates NEFA availability [42]. In our study of lipid metabolism, plasma adiponectin concentrations were independent of NEFA concentrations under conditions of hyperinsulinaemia in the range of postprandial values, suggesting that adiponectin does not play a major

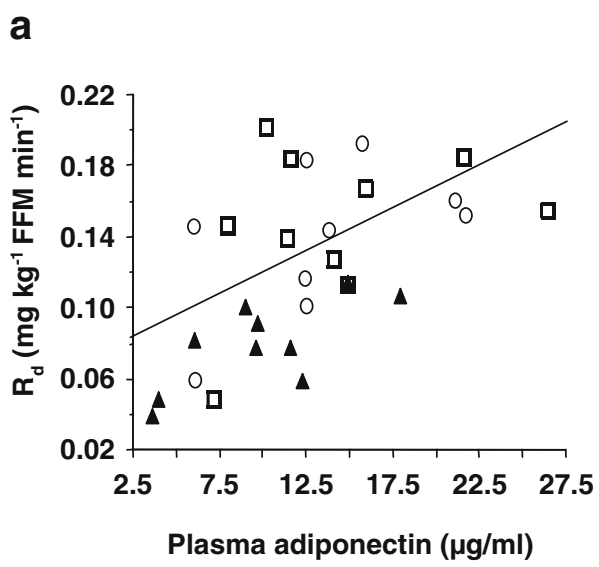

Fig. 5 Muscle glucose disposal rate in relation to adiponectin during pregnancy. Women were analysed longitudinally throughout pregnancy in basal state (overnight fast) and during euglycaemichyperinsulinaemic clamp. Muscle glucose disposal $\left(R_{d}\right)$ was assessed during insulin infusion in pregravid and pregnant states. role in the regulation of lipolysis. The rate of lipid oxidation was independent of adiponectin concentration under basal and insulin-stimulated conditions, although the ability of insulin to suppress fat oxidation in late pregnancy was clearly impaired (Table 3). A similar situation has been observed in obese individuals [43] and contrasts with in vitro data showing that adiponectin stimulates lipid oxidation through activation of AMP protein kinase [44]. One potential mechanism for the lack of regulation of fat oxidation in vivo may be increased production of adipocytokines [45]. During pregnancy, this could also be contributed to by the low-grade inflammatory situation originating in white adipose tissue and placenta through increased production of various cytokines including IL-6 and TNF- $\alpha$ [46, 47]. In addition, TNF- $\alpha$ is the best predictor of peripheral insulin resistance during pregnancy and, at the same time, is able to reduce adiponectin expression and secretion [45, 48]. Taking all of this into account, we view the pregnancy-mediated increase in TNF- $\alpha$ as an important functional link between hypoadiponectinaemia and insulin resistance. In the present study, the relationship between adiponectin and insulin sensitivity exclusively reflected changes of peripheral glucose fluxes in late pregnancy. This contrasts with studies reporting an association between basal and insulin-stimulated hepatic glucose production and adiponectin in situations of severe insulin resistance and may represent a feature that is specific to pregnancy $[28,29,49]$.

In conclusion, human pregnancy is a condition of hypoadiponectinaemia reflected at the level of the distribution of HMW adiponectin complexes. Assessing pregnancy as a model of insulin resistance with significant changes in fat mass but without obesity, our data emphasise the stronger involvement of adiponectin with regulation of glucose metabolism than with excess fat deposition. The pregnancy-mediated adiponectin changes are related to impairment of peripheral insulin sensitivity of glucose but not lipid metabolism. We propose that these changes are

b

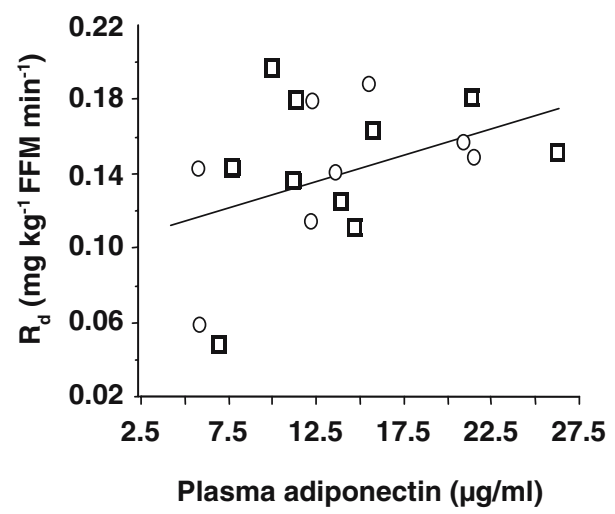

a Correlation with total plasma adiponectin (pre-gravid, early and late pregnancy). b Correlation with total plasma adiponectin omitting data in late pregnancy. Circles, pre-gravid; squares, early pregnancy; triangles, late pregnancy. $R=0.55 ; p=0.001$ (a); $R=0.41$; $p=0.08(\mathbf{b}) . F F M$, fat-free mass 
Table 3 Lipid metabolism under hyperinsulinaemic-euglycaemic steady-state conditions

\begin{tabular}{lccc}
\hline & Pregravid & Early pregnancy & Late pregnancy \\
\hline Lipid oxidation rate $\left(\mathrm{mg} \mathrm{kg}^{-1} \mathrm{FFM} \mathrm{min}^{-1}\right)$ & $-0.07 \pm 0.10^{\mathrm{b}}$ & $-0.08 \pm 0.10^{\mathrm{b}}$ & $0.36 \pm 0.11^{\mathrm{a}, \mathrm{b}}$ \\
Plasma NEFA (mmol/1) & $0.10 \pm 0.02^{\mathrm{b}}$ & $0.09 \pm 0.01^{\mathrm{a}, \mathrm{b}}$ & $0.15 \pm 0.02^{\mathrm{a}, \mathrm{b}}$ \\
\hline
\end{tabular}

Results are means $\pm \mathrm{SD}$ of ten subjects studied longitudinally before and during pregnancy. Differences statistically significant compared with non-pregnant with $p<0.001$

${ }^{\text {a}}$ Pregnant vs pregravid

${ }^{\mathrm{b}}$ Insulin vs basal

part of the complex metabolic adaptations of maternal tissues to pregnancy.

Acknowledgements This work was supported by NIH Grant RO1HD022965 and DAGC grant-in-aid 484-04.

\section{References}

1. Catalano PM, Wong WW, Drago NM, Amini SB (1995) Estimating body composition in late gestation: a new hydration constant for body density and total body water. Am J Physiol 268:E153-E158

2. Herrera E (2002) Lipid metabolism in pregnancy and its consequences in the fetus and newborn. Endocr Rev 19:43-55

3. Catalano PM, Roman-Drago N, Amini SB, Sims EAH (1998) Longitudinal changes in body composition and energy balance in lean women with normal and abnormal glucose tolerance during pregnancy. Am J Obstet Gynecol 179:156-165

4. Hauguel S, Gilbert M, Girard J (1987) Pregnancy-induced insulin resistance in liver and skeletal muscles in the conscious rabbit. Am J Physiol 252:E165-E169

5. Guerre-Millo M (2003) Extending the glucose/fatty acid cycle: a glucose/adipose tissue cycle. Biochem Soc Trans 6:1161-1164

6. Scherer PE, Williams S, Fogliano M, Baldini G, Lodish HF (1995) A novel serum protein similar to $\mathrm{C} 1 \mathrm{q}$, produced exclusively in adipocytes. J Biol Chem 270:26746-26749

7. Pajvani UB, Du X, Combs TP et al (2003) Structure-function studies of the adipocyte-secreted hormone Acrp30/adiponectin. Implications for metabolic regulation and bioactivity. J Biol Chem 278:9073-9085

8. Hu E, Liang P, Spiegelman BM (1996) AdipoQ is a novel adipose-specific gene dysregulated in obesity. J Biol Chem 271:10697-10703

9. Arita Y, Kihara S, Ouchi N et al (1999) Paradoxical decrease of an adipose-specific protein, adiponectin, in obesity. Biochem Biophys Res Commun 257:79-83

10. Tschritter O, Fritsche A, Thamer C et al (2003) Plasma adiponectin concentrations predict insulin sensitivity of both glucose and lipid metabolism. Diabetes 52:239-243

11. Combs TP, Berg AH, Rajala MW et al (2003) Sexual differentiation, pregnancy, calorie restriction, and aging affect the adipocyte-specific secretory protein adiponectin. Diabetes 52:268-276

12. Weyer C, Funahashi T, Tanaka S et al (2001) Hypoadiponectinemia in obesity and type 2 diabetes: close association with insulin resistance and hyperinsulinemia. J Clin Endocrinol Metab 86:1930-1935

13. Stefan N, Stumvoll M, Vozarova B et al (2003) Plasma adiponectin and endogenous glucose production in humans. Diabetes Care 26:3315-3319

14. Lara-Castro C, Luo N, Wallace P, Klein RL, Garvey WT (2006) Adiponectin multimeric complexes and the metabolic syndrome trait cluster. Diabetes 55:249-259
15. Fisher FF, Trujillo ME, Hanif W et al (2005) Serum high molecular weight complex of adiponectin correlates better with glucose tolerance than total serum adiponectin in Indo-Asian males. Diabetologia 48:1084-1087

16. Waki H, Yamauchi T, Kamon J et al (2003) Impaired multimerization of human adiponectin mutants associated with diabetes. Molecular structure and multimer formation of adiponectin. J Biol Chem 278:40352-40363

17. Carpenter MW, Coustan DR (1982) Criteria for screening tests for gestational diabetes. Am J Obstet Gynecol 144:768-773

18. Keys A, Brozek J (1953) Body fat in adult man. Physiol Rev 3:245-325

19. Steele R (1959) Influences of glucose loading and of injected insulin on hepatic glucose output. Ann N Y Acad Sci 82: 420-430

20. DeFronzo RA, Tobin D, Andres R (1979) Glucose clamp technique: a method for quantifying insulin secretion and resistance. Am J Physiol 237:E214-E223

21. Tserng KY, Kalhan SC (1983) Calculation of substrate turnover rate in stable isotope tracer studies. Am J Physiol 245: E308-E311

22. Frayn KN (1983) Calculation of substrate oxidation rates in vivo from gaseous exchange. J Appl Physiol 55:628-634

23. Laemmli UK (1970) Cleavage of structural proteins during the assembly of the head of bacteriophage T4. Nature 227:680-685

24. Bobbert T, Rochlitz H, Wegewitz U et al (2005) Changes of adiponectin oligomer composition by moderate weight reduction. Diabetes 54:2712-2719

25. Cseh K, Baranyi E, Melczer Z, Kaszas E, Palik E, Winkler G (2004) Plasma adiponectin and pregnancy-induced insulin resistance. Diabetes Care 27:274-275

26. Worda C, Leipold H, Gruber C, Kautzky-Willer A, Knofler M, Bancher-Todesca D (2004) Decreased plasma adiponectin concentrations in women with gestational diabetes mellitus. Am J Obstet Gynecol 191:2120-2124

27. Kondo E, Sugiyama T, Kusaka H, Toyoda N (2004) Adiponectin mRNA levels in parametrial adipose tissue and serum adiponectin levels are reduced in mice during late pregnancy. Horm Metab Res 36:465-469

28. Reeds DN, Yarasheski KE, Fontana L et al (2006) Alterations in liver, muscle, and adipose tissue insulin sensitivity in men with HIV infection and dyslipidemia. Am J Physiol 290: E47-E53

29. Bugianesi E, Pagotto U, Manini R et al (2005) Plasma adiponectin in nonalcoholic fatty liver is related to hepatic insulin resistance and hepatic fat content, not to liver disease severity. J Clin Endocrinol Metab 3498-3504

30. Ehrenberg HM, Huston-Presley L, Catalano PM (2003) The influence of obesity and gestational diabetes mellitus on accretion and the distribution of adipose tissue in pregnancy. Am J Obstet Gynecol 189:944-948

31. Thamer C, Machann J, Tschritter O et al (2002) Relationship between serum adiponectin concentration and intramyocellular lipid stores in humans. Horm Metab Res 34:646-649

32. Qiao L, Maclean PS, Schaack J et al (2005) C/EBPalpha regulates human adiponectin gene transcription through an intronic enhancer. Diabetes 54:1744-1754 
33. Fasshauer M, Kralisch S, Klier M et al (2003) Adiponectin gene expression and secretion is inhibited by interleukin- 6 in 3T3-L1 adipocytes. Biochem Biophys Res Commun 301:1045-1050

34. Degawa-Yamauchi M, Moss KA, Bovenkerk JE et al (2005) Regulation of adiponectin expression in human adipocytes: effects of adiposity, glucocorticoids, and tumor necrosis factor alpha. Obes Res 13:662-669

35. Brame LA, Considine RV, Yamauchi M, Baron AD, Mather KJ (2005) Insulin and endothelin in the acute regulation of adiponectin in vivo in humans. Obes Res 13:582-588

36. Combs TP, Berg AH, Obici S, Scherer PE, Rossetti L (2001) Endogenous glucose production is inhibited by the adiposederived protein Acrp 30. J Clin Invest 108:1875-1881

37. Motoshima H, Wu X, Sinha MK, Hardy VE et al (2002) Differential regulation of adiponectin secretion from cultured human omental and subcutaneous adipocytes: effects of insulin and rosiglitazone. J Clin Endocrinol Metab 87:5662-5667

38. Pajvani UB, Hawkins M, Combs TP et al (2004) Complex distribution, not absolute amount of adiponectin, correlates with thiazolidinedione-mediated improvement in insulin sensitivity. J Biol Chem 279:12152-12162

39. Tsao TS, Tomas E, Murrey HE et al (2003) Role of disulfide bonds in Acrp30/adiponectin structure and signaling specificity. Different oligomers activate different signal transduction pathways. J Biol Chem 278:50810-50817

40. Bruce C, Bumby J, Mangnall D, Fraser RB (1992) Evidence that the insulin resistance of pregnancy may not involve a postreceptor defect in human adipocytes. Diabetes Res Clin Pract $16: 75-84$
41. Yamauchi T, Kamon J, Minokoshi Y et al (2002) Adiponectin stimulates glucose utilization and fatty-acid oxidation by activating AMP-activated protein kinase. Nat Med 8:1288-1295

42. Bernstein EL, Koutkia P, Ljungquist K, Breu J, Canavan B, Grinspoon S (2004) Acute regulation of adiponectin by free fatty acids. Metabolism 53:790-793

43. Stefan N, Vozarova B, Funahashi T et al (2002) Plasma adiponectin levels are not associated with fat oxidation in humans. Obes Res 10:1016-1020

44. Tomas E, Tsao TS, Saha AK et al (2002) Enhanced muscle fat oxidation and glucose transport by ACRP30 globular domain: acetyl-CoA carboxylase inhibition and AMP-activated protein kinase activation. Proc Natl Acad Sci U S A 99:16309-16313

45. Dietze-Schroeder D, Sell H, Uhlig M, Koenen M, Eckel J (2005) Autocrine action of adiponectin on human fat cells prevents the release of insulin resistance-inducing factors. Diabetes 54:2003-2011

46. Radaelli T, Varastehpour A, Catalano P, Hauguel-de Mouzon S (2003) Gestational diabetes induces placental genes for chronic stress and inflammatory pathways. Diabetes 52:2951-2958

47. Radaelli T, Uvena-Celebrezze J, Minium J, Huston-Presley L, Catalano P, Hauguel-de Mouzon S (2006) Maternal interleukin6: marker of fetal growth and adiposity. J Soc Gynecol Invest 13:53-57

48. Kirwan JP, Hauguel-de Mouzon S, Lepercq J et al (2002) TNFalpha is a predictor of insulin resistance in human pregnancy. Diabetes 51:2207-2213

49. Bajaj M, Suraamornkul S, Piper P et al (2004) Decreased plasma adiponectin concentrations are closely related to hepatic fat content and hepatic insulin resistance in pioglitazone-treated type 2 diabetic patients. J Clin Endocrinol Metab 89:200-206 